\title{
DATA MANAGEMENT SYSTEMS FOR SCIENTIFIC APPLICATIONS
}

\author{
Reagan W. Moore \\ San Diego Supercomputer Center \\ San Diego, CA, USA
}

\begin{abstract}
Scientific applications now have data management requirements that extend beyond the local computing environment. Scientific disciplines are assembling data collections that represent the primary source data for current research. SDSC has developed data management systems to facilitate use of published digital objects. The associated infrastructure includes persistent archives for managing technology evolution, data handling systems for collection-based access to data, collection management systems for organizing information catalogs, digital library services for manipulating data sets, and data grids for federating multiple collections. The infrastructure components can be characterized as interoperability systems for digital object management, information management, and knowledge management. Examples of the application of the technology include distributed collections and data grids for astronomical sky surveys, high energy physics data collections, and art image digital libraries.
\end{abstract}

Keywords: Data handling systems, digital libraries, grids, persistent archives

\section{INTRODUCTION}

Scientific disciplines are starting to assemble primary source data for use by researchers [7]. The data are typically distributed across multiple administration domains and are stored on heterogeneous storage systems. The challenge is to facilitate the organization of these primary data resources into collections without compromising local control. At the same time, middleware is needed to support uniform access to the data sets, including APIs for direct application discovery and manipulation of the data, command line interfaces for accessing data sets from scripts, and web GUIs for interactive browsing and presentation of data sets. 
The development of infrastructure to support the publication of scientific data must recognize that information repositories and knowledge bases are also needed. One can differentiate between infrastructure components that provide:

- Data storage of digital objects that are either simulation output or remote sensing data. The digital objects are representations of reality, generated either through a hardware remote sensing device or by execution of an application.

- Information repositories that store attributes about the digital objects. The attributes are typically stored as metadata in a catalog or database.

- Knowledge bases that characterize relationships between sets of metadata. An example is rule-based ontology mapping [6] that provides the ability to correlate information stored in multiple metadata catalogs.

A scientific data publication system will need to support ingestion of digital objects, querying of metadata catalogs to identify objects of interest, and integration of responses across multiple information repositories. Fortunately, a rapid convergence of information management technology and data handling systems is occurring for the support of scientific data collections. The goal is to provide mechanisms for the publication of scientific data for use by an entire research community. The approach used at the San Diego Supercomputer Center is to organize distributed data sets through creation of a logical collection [9]. The ownership of the data sets is assigned to the collection, and a data handling system is used to create, move, copy, replicate, and read collection data sets. Since all accesses to the collection data sets are done through the data handling system, it then becomes possible to put the data sets under strict management control, and implement features such as access control lists, usage audit trails, replica management, and persistent identifiers.

Effectively, a distributed collection can be created in which the local resources remain under the control of the local site, but the data sets are managed by the global logical collection. Researchers authenticate themselves to the collection, and the collection in turn authenticates itself to the distributed storage systems on which the data sets reside. The collection manages the access control lists for each data set independently of the local site. The local resources are effectively encapsulated into a collection service, removing the need for researchers to have user accounts at each site where the data sets are stored. 
The data handling system serves as an interoperability mechanism for managing storage systems. Instead of directly storing digital objects in an archive or file system, the interposition of a data handling system allows the creation of a collection that spans multiple storage systems. It is then possible to automate the creation of a replica in an archival storage system, cache a copy of a digital object onto a local disk, and support the remote manipulation of the digital object. The creation of data handling systems for collection-based access to published scientific data sets makes it possible to automate all data management tasks. In turn, this makes it possible to support data mining against collections of data sets, including comparisons between simulation and measurement, and statistical analysis of the properties of multiple data sets. Data set handling systems can be characterized as interoperability mechanisms that integrate local data resources into global resources. The interoperability mechanisms include

- inter-domain authentication,

- transparent protocol conversion for access to all storage systems,

- global persistent identifiers that are location and protocol independent,

- replica management for cached and archived copies,

- container technology to optimize archival storage performance and co-locate small data sets, and

- tools for uniform collection management across file systems, databases, and archives.

\section{DATA HANDLING INFRASTRUCTURE}

The data management infrastructure is based upon technology from multiple communities that are developing archival storage systems, parallel and XML [3] database management systems, digital library services, distributed computing environments, and persistent archives. The combination of these systems is resulting in the ability to describe, manage, access, and build very large distributed scientific data collections. Several key factors are driving the technology convergence:

- Development of an appropriate information exchange protocol and information tagging model. The ability to tag the information content makes it possible to directly manipulate information. The 
eXtensible Markup Language (XML) provides a common information model for tagging data set context and provenance. Document Type Definitions (and related organizational methods such as XML Schema [10]) provide a way to organize the tagged attributes. Currently, each discipline is developing their own markup language (set of attributes) for describing their domain-specific information. The library community has developed some generic attribute sets such as the Dublin core to describe provenance information. The combination of the Dublin core metadata and discipline specific metadata can be used to describe scientific data sets.

- Differentiation between the physical organization of a collection (conceptually the table structures used to store attributes in object-relational databases) and the logical organization of a collection (the schema). If both contexts are published, it becomes possible to automate the generation of the SQL commands used to query relational databases. For XML-based collections, the emergence of XML Matching and Structuring languages [1] makes it possible to construct queries based upon specification of attributes within XML DTDs. Thus attribute-based identification of data sets no longer requires the ability to generate SQL or XQL commands from within an application.

- Differentiation of the organization and access mechanisms for a logical collection from the organization and access mechanisms required by a particular storage system. Conceptually, data handling systems store data in storage systems rather than storage devices. By keeping the collection context independent of the physical storage devices, and providing interoperability mechanisms for data movement between storage systems, logical data set collections can be created across any type of storage system. Existing data collections can be transparently incorporated into the logical collection. The only requirement is that the logical collection be given access control permissions for the local data sets. The data handling system becomes the unifying middleware for access to distributed data sets.

- Differentiation of the management of information repositories from the storage of metadata into a catalog. Information management systems provide the ability to manage databases. It is then possible to migrate metadata catalogs between database instantiations, extend the schema used to organize the catalogs, and export metadata as XML or HTML formatted files. 
The ability to manipulate data sets through collection-based access mechanisms enables the federation of data collections and the creation of persistent archives. Federation is enabled by publishing the schema used to organize a collection as an XML DTD. Information discovery can then be done through queries based upon the semi-structured representation of the collection attributes provided by the XML DTD. Distributed queries across multiple collections can be accomplished by mapping between the multiple DTDs, either through use of rules-based ontology mapping, or token-based attribute mapping.

Persistent archives can be enabled by archiving the context that defines both the physical and logical collection organization along with the data sets that comprise the collection [8]. The collection context can then be used to recreate the collection on new database technology through an instantiation program. This makes it possible to migrate a collection forward in time onto new technology. The collection description is instantiated on the new technology, while the data sets remain on the physical storage resource. The collection instantiation program is updated as database technology evolves, while the archived data remains under the control of the data handling system. As the archive technology evolves, new drivers are added to the data handling system to interoperate with the new data access protocols.

The implementation of information management technology needs to build upon the information models and manipulation capabilities that are coming from the Digital Library community, and the remote data access and procedure execution support that is coming from the distributed computing community. The Data Access Working Group of the Grid Forum [5] is promoting the development of standard implementation practices for the construction of grids. Grids are inherently distributed systems that tie together data, compute, and visualization resources. Researchers rely on the grid to support all aspects of information management and data manipulation. An end-to-end system provides support for:

- Knowledge discovery - ability to identify relationships between digital objects stored in different discipline collections

- Information discovery - ability to query across multiple information repositories to identify data sets of interest

- Data handling - ability to read data from a remote site for use within an application

- Remote processing - ability to filter or subset a data set before transmission over the network 
- Publication - ability to add data sets to collections for use by other researchers

- Analysis - ability to use data in scientific simulations, or for data mining, or for creation of new data collections

These services are implemented as middleware that hide the complexity of the diverse distributed heterogeneous resources that comprise data and compute grids [4]. The services provide four key functionalities or transparencies that simplify the complexity of accessing distributed heterogeneous systems.

- Name transparency - Unique names for data sets are needed to guarantee a specific data set can be found and retrieved. However, it is not possible to know the unique name of every data set that can be accessed within a data grid (potentially billions of objects). Attribute based access is used so that any data set can be identified either by data handling system attributes, or Dublin core provenance attributes, or discipline specific attributes.

- Location transparency - Given the identification of a desired data set, a data handling system manages interactions with the possibly remote data set. The actual location of the data set can be maintained as part of the data handling system attributes. This makes it possible to automate remote data access. When data sets are replicated across multiple sites, attribute-based access is essential to allow the data handling system to retrieve the "closest" copy.

- Protocol transparency - Data grids provide access to heterogeneous data resources, including file systems, databases, and archives. The data handling system can use attributes stored in the collection catalog to determine the particular access protocol required to retrieve the desired data set. For heterogeneous systems, servers can be installed on each storage resource to automate the protocol conversion. Then an application can access objects stored in a database or in an archive through a uniform user interface.

- Time transparency - At least five mechanisms can be used to minimize retrieval time for distributed objects: data caching, data replication, data aggregation, parallel $\mathrm{I} / \mathrm{O}$, and remote data filtering. Each of these mechanisms can be automated as part of the data handling system. Data caching can be automated by having the data handling system pull data from the remote archive to a local data cache. Data replication across multiple storage resources 
can be used to minimize wide area network traffic. Data aggregation through the use of containers can be used to minimize access latency to archives or remote storage systems. Parallel I/O can be used to minimize the time needed to transfer a large data set. Remote data filtering can be used to minimize the amount of data that must be moved. This latter capability requires the ability to support remote procedure execution at the storage resource.

\section{APPLICATION}

A collection-based data management system has the following software infrastructure layers:

- Data Grid - for federation of access to multiple data collections and digital libraries

- Digital library - provide services for discovering, manipulating, and presenting data from collections

- Data collection - provide support for extensible, dynamically changing organizations of data sets

- Data handling system - provide persistent IDs for collection-based access to data sets

- Persistent archive - provide collection-based storage of data sets, with the ability to handle evolution of the software infrastructure.

The essential infrastructure component is the data handling system. It is possible to use data handling systems to assemble distributed data collections, integrate digital libraries with archival storage systems, federate multiple collections into a data grid, and create persistent archives. An example that encompasses all of these cases is the 2-Micron All Sky Survey image archive. The 2MASS survey is an astronomy project led by the University of Massachusetts and Caltech to assemble a catalog of all stellar objects that are visible at the 2 -micron wavelength. The goal of the project is to provide a catalog that lists attributes of each object, such as brightness and location. The final catalog can contain as many as 2 billion stars and 200 million galaxies. Of interest to astronomers is the ability to analyze the images of all the galaxies. This is a massive data analysis problem since there will be a total of 5 million images comprising 10 terabytes of data.

A collaboration between IPAC at Caltech and the NPACI program at SDSC is building an image catalog of all of the 2MASS observations. A digital library is being created at Caltech that records which image 
contains each galaxy. The images are sorted into 147,000 containers to co-locate all images from the same area in the sky. The image collection is then replicated between two archives to provide disaster recovery. The SDSC Storage Resource Broker [2] is used as the data handling system to provide access to archives at Caltech and SDSC where the images are replicated.

The usage model supports the following access scenario:

- Astronomers access the catalog at Caltech to identify galaxy types of interest.

- Digital library procedures determine which images need to be retrieved.

- The data handling system maps the image to the appropriate container, retrieves the container from the archive, and caches the container on a disk.

- The desired image is then read from the disk and returned to the user through the digital library.

Since the images are accessed through the data handling system, the desired images can be retrieved from either archive depending upon load or availability. If the container has already been migrated to disk cache, the data handling system can immediately retrieve the image from disk avoiding the access latency inherent in reading from the archive tape system. If one archive is inaccessible, the data handling system automatically defaults to the alternate storage system. If data is migrated to alternate storage systems, the persistent identifier remains the same and the data handling system adds the location and protocol access metadata for the new storage system. The system incorporates persistent archive technology, data handling systems, collection management tools, and digital library services in order to support analysis of galactic images.

Given the ability to access a 10-terabyte collection that contains a complete sky survey at the 2-micron wavelength, it is then possible to do data intensive analyses on terascale computer platforms . The data rates that are supported by a teraflops-capable computer will be over 100 Megabytes per second. It will be possible to read the entire collection in 30 hours. During this time period, over ten billion operations can be done on each of the five million images. Effectively, the entire survey can be analyzed in a "typical" computation on a teraflops computer. 


\section{SUMMARY}

Data intensive computing is facilitated by the organization of scientific data into collections that can then be processed by the scientific community. In the long run, the utility of scientific computation will be measured by the publication of the results of the computation into collections for use by the rest of the community. Digital objects that remain as local files on a researcher's workstation will be of little use to the scientific discipline. The utility of digital objects will be directly related to the specification of their context through membership in a scientific data collection. At this point, the fundamental access paradigm will shift to reading and writing data from collections, with applications using APIs to discover, access, and manipulate collection-based data. Each discipline will use their data repositories, information catalogs, and knowledge bases to provide direct access to all of the primary data sources for their domain.

\section{Acknowledgments}

The topics presented in this report were developed by the Data Intensive Computing Environment Group at the San Diego Supercomputer Center. DICE group members include Chaitan Baru, Arcot Rajasekar, Amarnath Gupta, Bertram Ludaescher, Richard Marciano, Michael Wan, and Ilya Zaslavsky. The data management technology has been developed through multiple federally sponsored projects, including DARPA project F19628-95-C-0194 "Massive Data Analysis Systems," the DARPA/USPTO project F19628-96-C-0020 "Distributed Object Computation Testbed," the Data Intensive Computing thrust area of the NSF project ASC 9619020 "National Partnership for Advanced Computational Infrastructure," the NASA Information Power Grid project, and the DOE ASCI/ASAP project "Data Visualization Corridor." The development of persistent archives was supported by a NARA extension to the DARPA/USPTO Distributed Object Computation Testbed, project F19628-96-C-0020.

\section{References}

[1] Baru, C., V. Chu, A. Gupta, B. Ludascher, R. Marciano, Y. Papakonstantinou, and P. Velikhov. XML-Based Information Mediation for Digital Libraries. In ACM Conf. On Digital Libraries, Berkeley, CA, 1999. Exhibition program.

[2] Baru, C., R, Moore, A. Rajasekar, M. Wan, "The SDSC Storage Resource Broker," Proc. CASCON'98 Conference, November 30 December 3, 1998, Toronto, Canada. 
[3] Extensible Markup Language (XML). http://www.w3.org/XML/, 1998.

[4] Foster, I., and C. Kesselman. The Grid: Blueprint for a New Computing Infrastructure, Chapter 5, "Data Intensive Computing," Morgan Kaufmann, San Francisco, 1999.

[5] Grid Forum Remote Data Access Working Group. http://www.sdsc.edu/GridForum/RemoteData/

[6] Ludäscher, B., A. Gupta, M. E. Martone. Model-Based Information Integration in a Neuroscience Mediator System, demonstration track, 26th Intl. Conference on Very Large Databases (VLDB), Cairo, Egypt, September, 2000.

[7] Moore, R., C. Baru, P. Bourne, M. Ellisman, S. Karin, A. Rajasekar, S. Young, "Information Based Computing," Proceedings of the Workshop on Research Directions for the Next Generation Internet, May, 1997.

[8] Moore, R., C. Baru, A. Rajasekar, B. Ludascher, R. Marciano, M. Wan, W. Schroeder, and A. Gupta. Collection-Based Persistent Digital Archives - Part 1, D-Lib Magazine, March 2000, http://www.dlib.org/

[9] NPACI Data Intensive Computing Environment thrust area, http://www.npaci.edu/DICE/

[10] XML Schema, Working Draft. http://www .w3.org/TR/xmlschema$\{1,2\}$, September 2000. 


\section{DISCUSSION}

\section{Speaker: Reagan Moore}

Richard Fateman : Who pays for storage and access of these collections? How is annotation linked to data?

Reagan Moore : The collections are the result of community-wide efforts within scientific domains. An example is the National Virtual Observatory, which plans the integration of the major all sky survey image collections. The funding will come from their sponsoring agencies, including NSF and NASA. The users of the collections will be astronomers. Access to collections is supported through an application programmer's interface, a set of Unix command-line utilities, and a Java/NT browser. The NT browser can be executed from a CDROM, making it possible to load a client in a few minutes. The SDSC Storage Resource Broker (SRB) client runs on Linux, Unix workstations, Windows NT, and supercomputers.

David W. Walker : To what extent are the storage and data management systems you have described in production use by the end-user scientific community?

Reagan Moore : The major uses of the SDSC SRB are scientific communities that are assembling discipline-wide scientific collections. The largest production system is for the 2MASS two-micron all sky survey. Over 3 million images (6 terabytes of data) have been ingested into a collection, with the complete image set to be imported by January 2001. The SRB is installed at 35 sites, and has over 150 registered users.

Fred Gustavson : My question concerns efficiency. What scientific methods are being used by your systems for identifying and classifying data so that it is well organized to perform a large scientific computation on that data. An example would be the computational fluid dynamics computation you spoke about on historical data using new computational algorithms for computational fluid dynamics.

Reagan Moore : The organization of digital objects into a collection depends upon consensus within a scientific domain for the appropriate metadata. In the use of the Data Assimilation Office, the input data sets are observed data that have been organized for a daily analysis. The knowledge needed to use the data is embedded in the application. A simple unique label for each data object is the date of the assimilation run. An analysis requires retrieval and use of each data set for the time period of interest. More general characterizations are needed when the collection must support multiple applications. In particular, as new applications are developed, additional metadata attributes may be needed. For any collection it may be necessary to extend the schema to include 
the new attributes. Current technology supports the export and import of metadata from databases using XML DTDs, making this easy to do. Margaret Wright : What is the role of data compression? Where does it fit within the overall structure that you described?

Reagan Moore : Data compression can give compression factors of 1.5 for lossless algorithms, and factors of 10-100 for lossy algorithms. In practice, thumbnail (browse) images are created to help with discovery, and then the high resolution, lossless data objects are accessed. Compression can be supported at the remote site through proxy procedures. 